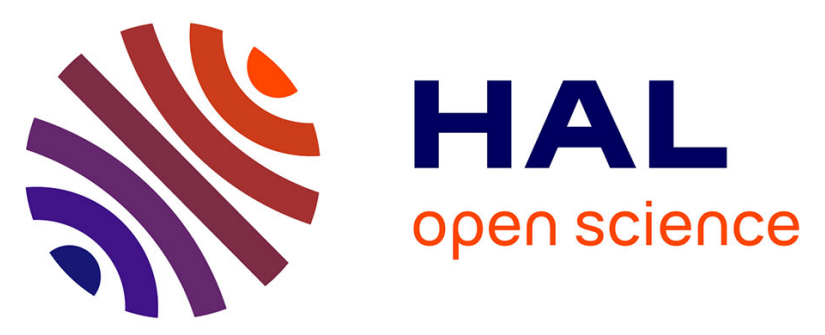

\title{
Design and fabrication of a single-mode and ultra-low loss hollow-core fiber based on Kagome-tubular hybrid lattice
}

F Amrani, J H Osório, F Delahaye, F Giovanardi, K Vasko, L Vincetti, B Debord, Frédéric Gérôme, F Benabid

\section{To cite this version:}

F Amrani, J H Osório, F Delahaye, F Giovanardi, K Vasko, et al.. Design and fabrication of a single-mode and ultra-low loss hollow-core fiber based on Kagome-tubular hybrid lattice. CLEO: Conference on Lasers and Electro-Optics 2021 US, May 2021, San José, United States. pp.STu1Q.2. hal-03285519

\section{HAL Id: hal-03285519 \\ https://hal.science/hal-03285519}

Submitted on 21 Jul 2021

HAL is a multi-disciplinary open access archive for the deposit and dissemination of scientific research documents, whether they are published or not. The documents may come from teaching and research institutions in France or abroad, or from public or private research centers.
L'archive ouverte pluridisciplinaire HAL, est destinée au dépôt et à la diffusion de documents scientifiques de niveau recherche, publiés ou non, émanant des établissements d'enseignement et de recherche français ou étrangers, des laboratoires publics ou privés. 


\title{
Design and fabrication of a single-mode and ultra-low loss hollow-core fiber based on Kagome-tubular hybrid lattice
}

\author{
F. Amrani ${ }^{1,2}$, J. H. Osório ${ }^{1}$, F. Delahaye ${ }^{1,2}$, F. Giovanardi' ${ }^{3}$, K. Vasko ${ }^{1}$, L. Vincetti ${ }^{3}$, B. Debord ${ }^{1,2}$, F. Gérôme ${ }^{1,2}$, \\ F. Benabid ${ }^{1,2}$ \\ ${ }^{I}$ GPPMM Group, XLIM Institute, CNRS UMR 7252, University of Limoges, Limoges, France \\ ${ }^{2}$ GLOphotonics, 123 Avenue Albert Thomas, Limoges, France \\ ${ }^{3}$ Department of Engineering 'Enzo Ferrari', University of Modena and Reggio Emilia, Modena, Italy \\ f.benabid@xlim.fr
}

\begin{abstract}
We propose a hybrid Kagome-tubular lattice hollow-core fiber for ultra-low loss and single-mode operation. The fiber displays a minimum loss of $1.6 \mathrm{~dB} / \mathrm{km}$ at $1050 \mathrm{~nm}$ and a higherorder modes extinction of $47 \mathrm{~dB}$ for a 10m-long fiber. (C) 2020 The Author(s)
\end{abstract}

\section{Introduction}

The recently demonstrated outstanding performances of Inhibited-Coupling (IC) hollow-core photonic-crystal fibers (HCPCFs) afford them a prominent position in state-of-the-art photonics and allot them great interest from the optical community. However, although the efforts on lessening the loss levels in HCPCFs have entailed impressive results, the challenge of combining a low loss, single-mode (SM), and polarization-maintaining HCPCF persists.

In IC fibers, even though there is ubiquitous presence of higher-order modes (HOMs), one can verge on a robust SM operation if the loss extinction ratio between the fiber lowest loss mode and the second-lowest loss mode is appropriately high. This approach can be successfully accomplished, for example, in six-tube (6T) single-ring tubular-lattice (SR-TL) HCPCFs, where an adequate ratio between the core and lattice tubes diameters provides effective refractive index matching between the core $\mathrm{LP}_{11}$-like modes (characteristically the main contaminating HOM in the fiber modal content) and the fundamental air-mode of the lattice tubes [1-3]. The confinement loss (CL) of 6T SR-TL HCPCF is, however, generally high. Other designs, such as nested-tubes HCPCFs, can offer much lower loss figures than 6T SR-TL HCPCF. Nevertheless, the ratio between the loss values of the fundamental and $\mathrm{LP}_{11}$-like core modes is considerably inferior to the ones achieved in 6T SR-TL HCPCFs (typically $20 \mathrm{~dB}$ for the nested-tubes design and higher than $30 \mathrm{~dB}$ for $6 \mathrm{~T}$ SR-TL HCPCFs $[3,4])$.

Here, we discourse on the design and fabrication of a new HCPCF, the hybrid Kagome-tubular lattice (HKT) HCPCF, which combines effective SM operation and ultralow CL. The cladding of the HKT HCPCF displays an inner cladding made of six isolated tubes, which confers the effective SM operation capability to the fiber, and a Kagome outer cladding, which allows decreasing the CL figures significantly. The experimentally achieved fiber exhibits a minimum loss of $1.6 \mathrm{~dB} / \mathrm{km}$ at $1050 \mathrm{~nm}$. Measurements on the fiber modal content showed a record HOM extinction ratio of $47 \mathrm{~dB}$ for a fiber length of $10 \mathrm{~m}$. We envisage that the HKT HCPCF design will grant a new route for the next-generation low-loss single-mode HCPCFs.

\section{Results}

Fig. 1a exposes the concept of the HKT HCPCF. The structure diagram is shown in Fig. 1a top part. The HKT HCPCF architecture exhibits an inner cladding made of six tubes and a Kagome outer cladding. The 6T cladding allows filtering the core $\mathrm{LP}_{11}$-like modes by resonant coupling between the latter and the fundamental mode of the cladding tubes. The Kagome cladding, in turn, allows to substantially decrease the CL. We firstly consider an ideal version of the HKT HCPCF where one assumes no physical connection between the inner and outer claddings. While unfeasible, this design allows studying the great potential of the HKT HCPCF to attain ultralow loss.

The capability of the HKT HCPCF to provide ultralow loss is shown in Fig. 1a (bottom), where the CL of different fiber designs (FD) is plotted for different normalized frequencies, $F=(2 t / \lambda)\left(n_{g}{ }^{2}-1\right)^{1 / 2}(t$ : cladding tubes thickness; $\lambda$ : wavelength; $n_{g}$ : glass refractive index). FD \#1, \#2, \#3, and \#4 represent, respectively, a jacketless tubular fiber, a SR-TL HCPCF, a Kagome fiber, and the ideal HKT HCPCF. Fig. 1a shows that the CL of HKT HCPCF is significantly lower than the CL of the other FDs. Noteworthily, the difference between the CL of FD \#4 and those of the other FDs reaches five orders of magnitude in the $2^{\text {nd }}$-order transmission band. It is remarkable that the ideal HKT HCPCF can achieve lower CL values than the loss of solid-core fibers (dashed line in Fig. 1a).

In a realizable version of the HKT HCPCF, thin tubes can be added to the structure to connect inner and outer claddings. The addition of the connecting tubes increases the loss of the ideal HKT HCPCF, still conserving its capability of providing ultralow loss. Fig. 1b compares the CL of state-of-the-art HCPCFs, namely SR-TL (I), 

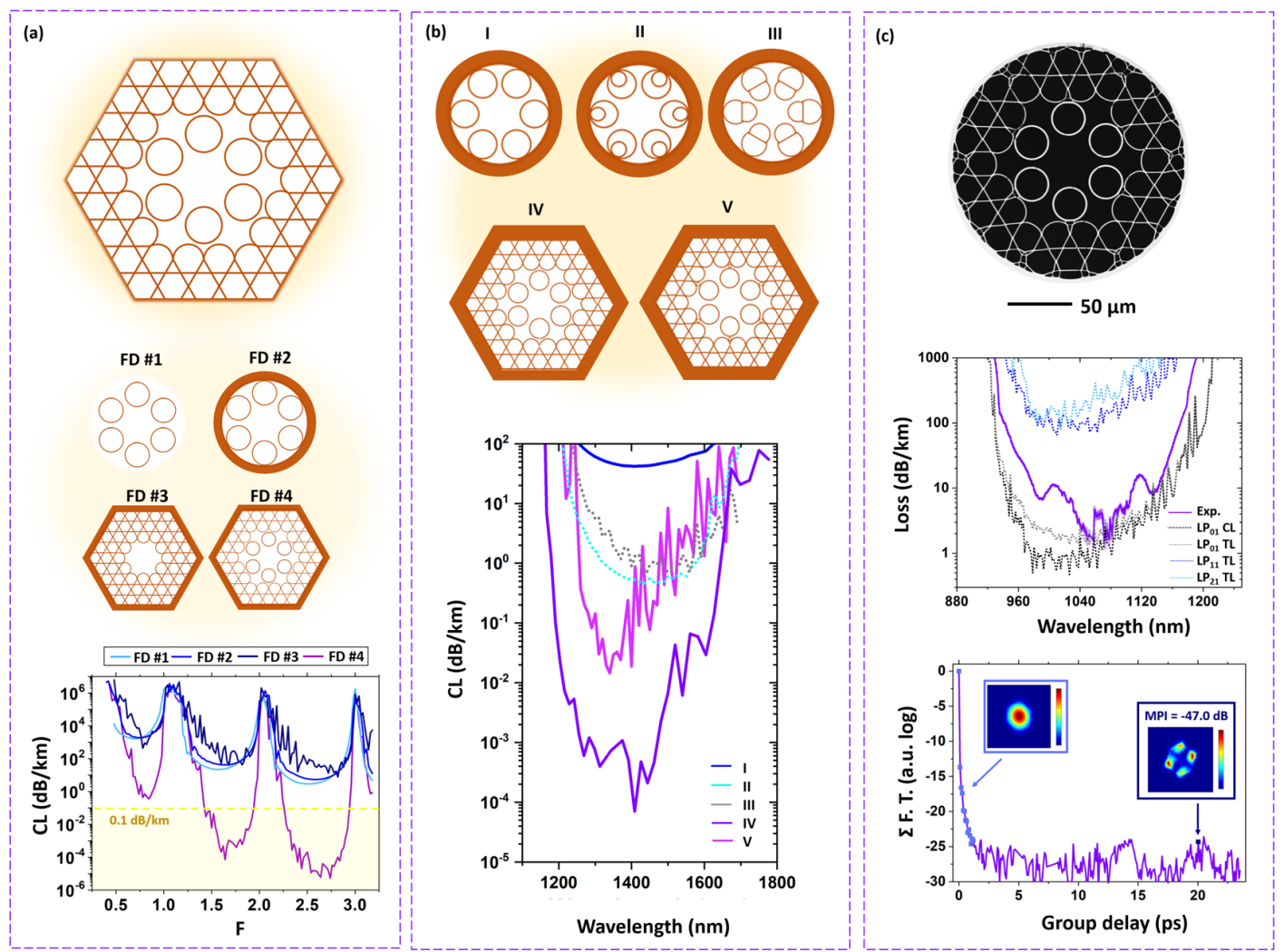

Fig. 1. (a) HKT HCPCF concept. CL for jacketless tubular (\#1), tubular (\#2), Kagome (\#3) and HKT (\#4) designs. (b) CL of state-of-the-art HCPCFs. (c) Experimental HKT HCPCF, measured and simulated loss for representative core modes; $\mathrm{S}^{2}$ measurement results for a $10 \mathrm{~m}$ fiber; MPI: multipath interference.

nested-tubes (II), and conjoined-tubes (III) with the ideal and the realizable HKT HCPCF (IV and V respectively). Remarkably, the HKT HCPCF has the potential of providing significantly lower loss values than the other FDs.

Fig. 1c exhibits the experimentally achieved HKT HCPCF. The thickness of the inner cladding tubes is $1.27 \mu \mathrm{m}$, and the core diameter of the fiber core measures $37.1 \mu \mathrm{m}$. The ratio between the diameters of the core and cladding tubes diameters $\left(D_{\text {tubes }} / D_{\text {core }}\right)$ has been optimized during fiber fabrication to attain optimal coupling between the core $\mathrm{LP}_{11}$ mode and the fundamental mode of the cladding tubes. In the experimental fiber, $D_{\text {tubes }} / D_{\text {core }}=0.62$.

Fig. 1c also exposes the measured loss, where a minimum value of $1.6 \mathrm{~dB} / \mathrm{km}$ has been obtained at $1050 \mathrm{~nm}$, and the calculated $\mathrm{CL}$ and total loss $(T L=C L+S S L$; SSL: surface scattering loss) of the core fundamental mode and the TL of representative HOM. Fig. 1c apprises that LP11-like and $L_{2}$-like modes have TL around two orders of magnitude larger than the TL of the fundamental core mode. Finally, we performed $\mathrm{S}^{2}$ measurements to evaluate the fiber modal content. At optimized coupling conditions, the $\mathrm{S}^{2}$ experiments showed a HOM extinction ratio of $47 \mathrm{~dB}$ for a $10 \mathrm{~m}$-long fiber (Fig. 1c bottom). Indeed, robust SM operation has been observed even when the input beam was misaligned. Measurements showed a HOM extinction of $24.7 \mathrm{~dB}$ when the input beam was offset by $10 \mu \mathrm{m}$ from its optimum position. The HKT HCPCF encompasses, therefore, low loss and robust SM operation.

\section{Conclusions}

We reported on a new HCPCF that combines low loss and effective SM operation. The novel design comprises a hybrid lattice with a $6 \mathrm{~T}$ inner cladding and a Kagome outer cladding. The experimental fiber showed a minimum loss of $1.6 \mathrm{~dB} / \mathrm{km}$ at $1050 \mathrm{~nm}$ and a $\mathrm{HOM}$ extinction ratio of $47 \mathrm{~dB}$ for a $10 \mathrm{~m}$-long fiber.

This research was funded through PIA program (grant $4 F$ ) and la region de la Nouvelle Aquitaine.

\section{References}

[1] Vincetti, L. \& Setti, V. Opt. Express 18, 23133-23146 (2010).

[2] Bradley, T. D. et al. J. Light. Technol. 31, 2752-2755 (2013).
[3] Uebel, P. et al. Opt. Lett. 41, 1951-1954 (2016).

[4] Jasion, G. T. et al. in OFC Conference, paper Th4B.4 (2020). 